\title{
RELIABILITY ANALYSIS OF SHELL TRUSS STRUCTURE BY HYBRID MONTE CARLO METHOD ${ }^{1}$
}

\author{
Beata Potrzeszcz-Sut \\ Kielce University of Technology, Kielce, Poland \\ e-mail: beatap@tu.kielce.pl
}

\begin{abstract}
The paper presents an example of reliability analysis of shell structures susceptible to stability loss from the condition of node snapping. In the reliability analysis of the structure, uncertain parameters of the task are represented by uncorrelated random variables. The approach used in the paper is an extension of the idea, which assumes the use of Neural Networks (NNs) in Monte Carlo (MC) simulations to analyze the reliability of the structure. For this purpose, it was necessary to build a simple hybrid system formed with the two independent sequentially working Finite Element Method (FEM) and Neural Networks applications.
\end{abstract}

Keywords: reliability, truss structure, Neural Networks, Hybrid Monte Carlo Method (HMC)

\section{Introduction}

Structures and structural elements should be designed so that they can be considered reliable throughout the entire expected lifetime under specified environmental conditions and for the assumed maintenance strategy, that is, that they meet the requirements regarding stability, load-bearing capacity and serviceability with a proper probability. Comprehensive information on the theoretical basis of structural reliability analysis can be found in extensive literature comprising thousands of monographs, dissertations and publications, cf. e.g. (Ditlevsen and Madsen, 1996; Gwóźdź and Machowski, 2011).

The problem of structural reliability analysis comes down to determining the probability of failure. As a standard, a deterministic approach is used during the design process, i.e. loads or material and geometric characteristics are treated as sets of multipliers and parameters. However, it should be emphasized that even the most accurate selection of these quantities may not guarantee safety of the structure. The reason for this situation is the uncertainty of the factor greater than that used in the design process according with the standards. In this case, calculations are made using probability theory, i.e. a probabilistic approach is used instead of a deterministic approach.

The work (JCSS, 1981) for the first time systematizes methods of reliability analysis, describes three levels of methods that clearly separate the deterministic from the probabilistic approaches: level I (Knauff, 2015), level II (Kunstmann et al., 2002), level III (Radoń, 2012; Dudzik, 2017).

In this work, one group of the above-mentioned methods was used, namely the Monte Carlo (MC) method, which represents a simulation approach to level III methods. Due to complexity of the data generation process, the MC simulation method was not widespread for many years. Currently, due to development of computer capabilities, this approach has become an important technique for analyzing reliability. Due to the basic disadvantage of the simulation approach by

\footnotetext{
${ }^{1}$ Article referring to the presentation at the 4th Polish Congress of Mechanics, 23rd International Conference on Computer Methods in Mechanics (PCM-CMM-2019), 8-12 September 2019, Kraków, Poland
} 
the classical MC method, which is a time-consuming computation, a hybrid approach is used in this paper. In this method, Artificial Neural Networks (NN) are used in Monte Carlo simulation. This procedure has been extensively developing in recent years (Pabisek, 2008; Kaliszuk, 2011).

Artificial Neural Networks (NN), due to their mode of action, are very effective in analyzing identification problems, cf. e.g. (Masters, 1996; Haykin, 1999). NNs are a very sophisticated modeling technique capable of mapping very complex functions. This paper uses one of the categories of neural networks, namely Feedforward Neural Network, commonly known as Back-Propagation Neural Network (BPNN). These networks are nonlinear, which causes that they are particularly often used in civil engineering issues. BPNN networks are a tool for universal approximation, which, when properly designed, are capable of modeling functions of almost any complexity. The big advantage of neural networks is the ability to simultaneously approximate several functions with the same arguments.

\subsection{Monte Carlo simulation}

The Monte Carlo (MC) method was developed and applied for the first time by mathematician of Polish origin Stanisław Ulam, and then the method was used by John von Neumann during World War II. The name Monte Carlo was created to indicate the random (gambling) nature of phenomena (Metropolis and Ulam, 1949). The Monte Carlo method has long been recognized as a technique with the highest accuracy among all probabilistic methods of analyzing structure reliability. Applications of the MC method in Civil Engineering issues are described in many papers, e.g. (Rubinstein and Kroese, 2008). The theoretical foundations of the Monte Carlo method can be presented based on information about the limit state of an engineering structure affected by uncertainty of its parameters. The probability of failure of the structure $p_{f}$ characterized by uncertainty of the parameters contained in the vector $\mathbf{X}$ can be presented in the form of an $n$ dimensional integral

$$
p_{f}=\operatorname{Prob}[G(\mathbf{X}) \leqslant 0]=\int_{G(\mathbf{X}) \leqslant 0} \ldots \int_{0} f_{X}(\mathbf{X}) d x
$$

where: $G(X)$ - limit state function of the structure, $f_{X}(\mathbf{X})$ - probability density function of the $n$ dimensional vector of random variables $\mathbf{X}$ describing the physical state of the structure.

Calculating integral (1.1) is a difficult task. The use of analytical methods to determine it is possible only in special cases. Due to efficiency, numerical integration methods are also of limited use.

A popular method of numerical calculation of multidimensional integrals is the Monte Carlo simulation method. It consists in generating realization of $x_{i}$ of $X$ variables, which are random parameters of the construction according to specific probability density distributions $f_{X}\left(x_{i}\right)$. In this way, single random samples are obtained $\widehat{x}_{i}$, which are used to validate the limit state of the structure. In the case of exceeding the limit state, i.e. when $G\left(\widehat{x}_{i}\right) \leqslant 0$, it is assumed that there was a structural failure. After putting through this experiment $N_{s}$ times, the probability of total failure can be calculated from the formula

$$
p_{f} \simeq \frac{n\left[G\left(\widehat{x}_{i}\right) \leqslant 0\right]}{N_{s}}
$$

where: $n\left[G\left(\widehat{x}_{i}\right) \leqslant 0\right]$ - number of simulations for which the structure has failed, $N_{s}$ - total number of attempts in the simulation.

The value of the number $N_{s}$ strongly affects the accuracy of the total failure probability estimation $p_{f}$, so it should be determined correctly. The $p_{f}$ value appraised by the Monte Carlo method is often called the estimated value or failure probability estimator. Very often in lite- 
rature, see paper (Olofsson and Andersson, 2012), a failure probability estimator is introduced, which is described by means of an indicator function (reliability indicator)

$$
\widetilde{p}_{f} \simeq \frac{1}{N_{s}} \sum_{i=1}^{N_{s}} I_{0 / 1}[G(\mathbf{X}) \leqslant 0]
$$

where the function indicator $I_{0 / 1}$ has the form

$$
I_{0 / 1}[G(\mathbf{X}) \leqslant 0]=\left\{\begin{array}{lll}
1 & \text { for } & G\left(X_{i}\right) \leqslant 0 \\
0 & \text { for } & G\left(X_{i}\right)>0
\end{array}\right.
$$

The accuracy of the estimation obtained using MC simulation can be determined by the formula $\widehat{e}_{M C}=\sqrt{\left(1-\widetilde{p}_{f}\right) /\left(N_{s} \widetilde{p}_{f}\right)}$ (Jelic et al., 2004).

The above formula shows that the error of estimation made using the MC method is approximately proportional to $1 / \sqrt{N_{s}}$ and does not depend on the dimension of the random variable $\mathbf{X}$. This is the main advantage of the method in engineering applications. According to standard (PN-EN 1993-1-1:2006), for the limit state of load capacity of the designed building, it is recommended to adopt a reliability index $\beta=3.8$. This value is equivalent to unreliability $\bar{p}_{f}=7.23 \cdot 10^{-5}$. To achieve sufficient accuracy in reliability analysis, a minimum number of simulations $N_{s}=9 \cdot 10^{7}$ is recommended (Rubinstein and Kroese, 2008).

Generating a large number of random samples $\widehat{x}_{i}$ needed to correctly estimate the probability of failure of the structure, $\bar{p}_{f}$ is a big limitation in using the classic Monte Carlo method. In some cases, the problem considered is very complicated and requires a long time to evaluate a single randomly selected parameter set. Therefore, numerical modifications of the classic MC method are used, see (Carpentier and Munos, 2012).

\subsection{Hybrid Monte Carlo method}

Hybrid reliability analysis methods are a relatively new approach using in the Monte Carlo simulations the so-called soft computation methods, which include neural networks. Due to the way $\mathrm{SN}$ is used, three main concepts can be pointed up.

1. Neural networks can replace polynomial functions of the limit state in approximation methods. This approach is presented by Deng et al. (2005), Dudzik and Potrzeszcz-Sut (2019).

2. The learning and testing set of the network are direct values of the probability of failure for the corresponding set values of the task parameters. An example of such an approach, the so-called expert system, is presented in the paper (Tsompanakis et al., 2005).

3. In the third group of methods, NN is used for generating samples in the Monte Carlo simulations. The patterns for network training and testing are computed by a FEM program. This approach was first proposed at work (Papadrakakis et al. 1996), for probabilistic analysis of the reliability of elasto-plastic frame constructions. In the following years, the hybrid method combining FEM and NN was successfully developed by independent groups: (Kaliszuk and Waszczyszyn, 2006; Pabisek et al., 2004; Pabisek, 2008; Papadopoulos et al., 2012).

In the presented work, the Hybrid Monte Carlo (HMC) method is used to analyze the reliability of elastic-plastic steel spatial trusses susceptible to loss of stability by snap-through of a node. For this purpose, the author's program implemented in the MATLAB computing environment was used. 
The course of action in the used hybrid approach is presented in the block diagram:

1. Defining the problem:

- adoption of random variables $\mathbf{X}$ and their probabilistic characteristics,

- defining the limit state function $G(\mathbf{X})$.

2. FEM simulation -- generating a learning and testing set of patterns for the neural network.

3. Preparation of the Neural Network -- design, learning and testing the NN in offline mode.

4. Simulation by Neural Network -- using NN to create a set for simulation by the MC method.

5. Reliability analysis:

- computation of the approximate value of the unreliability estimator $\bar{p}_{f}$ and reliability $q$,

- determination of reliability curves.

After defining the problem, i.e. adopting random variables, FEM simulation is performed, which allows one to generate the NN's learning and testing set. After the FEM simulation, the neural network formulation phase follows. The prepared $\mathrm{NN}$ is then used to generate the set applied in the MC simulation.

\section{Numerical examples}

The paper presents reliability analysis of two spatial trusses susceptible to stability loss from the condition of node snapping. The Newton-Raphson algorithm was used to determine the limit points on the equilibrium paths, in which both load and displacement control were used. The developed algorithm was implemented into own FEM program. In the performed nonlinear analysis, a two-node spatial truss finite element, which was described in (Waszczyszyn et al., 1994), was used.

In both examples, the reliability curves were determined using the Hybrid Monte Carlo method, see the diagram. The computations were made assuming three types of nonlinearity: NG - geometric nonlinearity, NF - physical nonlinearity, NGF - geometric and physical nonlinearity. The analyzed trusses, discussed later in the examples, were designed in accordance with the guidelines contained in standards (PN-EN 1990:2004; PN-EN 1993-1-1:2006; PN-EN 1993-1-4:2007), in such a way that individual bars do not undergo local buckling until reaching the limit point.

\subsection{Nonlinear material model}

For modeling material nonlinearities during the reliability analysis of steel spatial trusses, the power rule of Ramberg-Osgood (Ramberg and Osgood, 1943), was adopted

$$
\varepsilon=\left[\frac{\sigma}{\sigma_{Y}}+\alpha\left(\frac{\sigma}{\sigma_{Y}}\right)^{n}\right] \varepsilon_{0}, 2
$$

where: $\alpha=0.83$ - numerical parameter adopted according to (Pabisek, 2008), $n=5$ - exponent, adopted in accordance with standard (PN-EN 1993-1-4:2007).

In the case of nonlinear material characteristics, for NF and NGF cases, the initial modulus of elasticity $E$ occurring in the linear and displacement stiffness matrix (Waszczyszyn et al., 1994) 
must be replaced with the tangential module $E_{t}$ determined on the basis of the RO model. After computation of the stress $\sigma$, the tangential module $E_{t}$ can be determined

$$
E_{t}(\sigma)=\frac{1}{\left[\frac{1}{\sigma_{Y}}+\frac{n \alpha}{\sigma_{Y}}\left(\frac{\sigma}{\sigma_{Y}}\right)^{n-1}\right] \varepsilon_{0,2}}
$$

\subsection{Design, training and testing of Neural Networks}

Different variants of the analysis were considered, differing in the number of adopted random variables $\mathbf{X}=\left[X_{1}, X_{2}, \ldots, X_{N}\right]$. The detailed description of the variants is different for each example. The adopted variables were treated as elements of the input vector of networks. In each case, the output from the neural network was a scalar and corresponded to the limit load of the construction system $y=P_{u l t}$. For the adopted in this way input and output vectors, a network was designed, which realized the mapping $X_{i} \rightarrow y$.

After making initial computations, it was decided to continue using a one-way two-layer network with the Error Back-Propagation Learning Algorithm with architecture

$$
\text { BBNN: } N-H-1
$$

where: $N=3$ or 4 - number of network inputs, $H$ - the number of neurons in the hidden layer.

Bipolar activation functions for the hidden layer neurons and a linear activation function in the output neuron were assumed. SN formulation was done in the offline mode using the Neural Network Toolbox, working in MATLAB computing environment. The Levenberg-Marquard method was used to learn the network (Hagan and Menhaj, 1994). The SN formulation process was completed when the mean square errors of learning $M S E_{L}$ and testing $M S E_{T}$ adopted a value $\leqslant 1 \cdot 10^{-5}$.

For the hybrid simulation of the MC method, network architectures were selected that met the best the criterion of alignment of learning and testing errors.

\subsection{Example 1}

In this case, a not very rised lattice dome was analyzed, which is often presented in publications on nonlinear analysis and reliability, see works (Waszczyszyn et al., 1994; Radon, 2012).

(a)

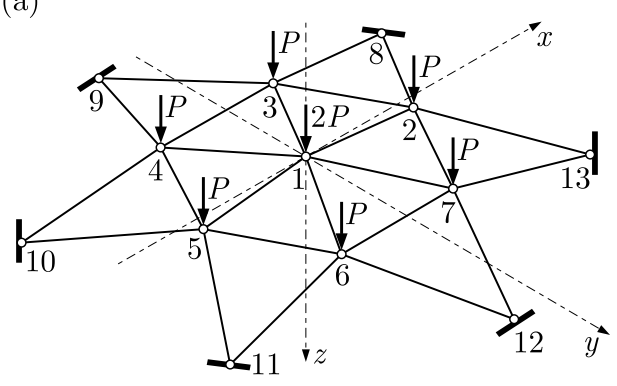

(b)

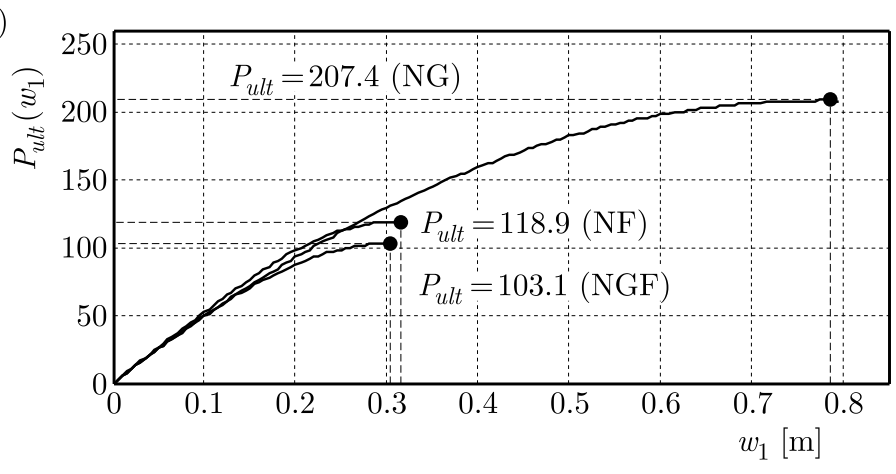

Fig. 1. Example 1: (a) spatial truss I: geometry and load diagram; (b) equilibrium paths determined for the vertical displacement of the node $w_{1}$ spatial truss, assuming average values of random variables

Figure 1a shows the diagram of the analyzed truss. The truss bars are made of RO168.3X10 round steel tubes with a modulus of elasticity $E=205 \mathrm{GPa}$ and a yield strength $\sigma_{Y}=235 \mathrm{MPa}$.

Figure $1 \mathrm{~b}$ shows examples of equilibrium paths $P\left(w_{1}\right)$ determined for vertical displacement $w_{1}$ of node 1 of the truss. As you we see, the values of the coordinates of the limit 
points at which the phenomenon of snap-through of the node occurs are different depending on the adopted type of analysis. These values are respectively:

$\mathrm{NG}-w_{1}^{G}=0.770 \mathrm{~m}, P_{u l t}=207.4 \mathrm{kN}$

$\mathrm{NF}-w_{1}^{G}=0.315 \mathrm{~m}, P_{u l t}=118.9 \mathrm{kN}$,

$\mathrm{NGF}-w_{1}^{G}=0.305 \mathrm{~m}, P_{u l t}=103.1 \mathrm{kN}$.

Reaching the limit point on the equilibrium path corresponds to the global loss of structure stability.

In this example, feedforward PBNN networks with one hidden layer, assuming the $N-H-1$ structure, were designed (see (2.3)). The entrance to the network were the following vectors:

$\mathrm{NG}-N=3, H=7, \mathbf{X}_{(3 \times 1)}=[E, A, P]$,

$\mathrm{NF}-N=4, H=7, \mathbf{X}_{(4 \times 1)}=\left[E, A, P, \sigma_{Y}\right]$,

$\mathrm{NGF}-N=4, H=10, \mathbf{X}_{(4 \times 1)}=\left[E, A, P, \sigma_{Y}\right]$,

with a hidden layer containing $H$ number of sigmoidal neurons and one linear output $y=P_{u l t}$.

\subsubsection{NG: geometric nonlinearity}

Table 1 presents the mean values $\mu_{i}$ and standard deviations $\sigma_{i}$ of the adopted random variables $\mathbf{X}=[E, A, P]$, which were selected from the ranges determined according to the three-sigma rule $X_{i} \in\left[\mu_{i}-3 \sigma_{i}, \mu_{i}+3 \sigma_{i}\right]$, see e.g. (Gwóźdź and Machowski, 2011). A coefficient of variation equal to $10 \%$ was assumed for each variable.

Tabel 1. Description of random variables for NG, NF and NGF models

\begin{tabular}{|c|c|c|}
\hline Random variable $X_{i}$ & Mean value $\mu_{i}$ & Standard deviation $\sigma_{i}$ \\
\hline \hline$X_{1}=E$ & $\mu_{E}=205 \mathrm{GPa}$ & $\sigma_{E}=20.5 \mathrm{GPa}$ \\
\hline$X_{2}=A$ & $\mu_{A}=0.049 \mathrm{~m}^{2}$ & $\sigma_{A}=0.0005 \mathrm{~m}^{2}$ \\
\hline$X_{3}=P$ & $\mu_{P}=1 \mathrm{kN}$ & $\sigma_{P}=0.1 \mathrm{kN}$ \\
\hline$X_{4}=\sigma_{Y}$ & $\mu_{f}=235 \mathrm{MPa}$ & $\sigma_{f}=23.5 \mathrm{MPa}$ \\
\hline
\end{tabular}

Table 2 presents random variables related to the considered cases. In case 1, three random variables were assumed to affect the load capacity of the analyzed truss. These variables are the modulus of elasticity $E$, the cross-sectional area of the elements $A$ and the load of the nodes of the system $P$. In rest cases $2-4$, the value of one of the variables was deterministic and was taken from the range belonging to the area of its variability. Reliability curves for cases 2-4 were created by performing MC simulation for each of the 30 accepted dividing points of the determined variable. 108 samples were drawn for each simulation, separately for each random variable according to the distribution given in Table 2 . Then, in virtue of the formulated SWPB: $3-7-1$ neural network, the limit load value was determined on the basis of which the value of indicator function (1.4), probability of failure (1.3) and then the reliability $\left(q=1-p_{f}\right)$ of the structure were determined.

Additionally, in each case, four versions (A, B, C, D) of the hybrid Monte Carlo simulation were considered, differing in the adopted probability distribution functions (PDFs) of the selected random variable. In Table 2, the letter $\mathrm{L}$ denotes the log-normal distribution, and the letter $\mathrm{N}$ the normal distribution of random variables, and "det." is the abbreviation for the determined value.

In this way, 14 reliability curves were determined, presented in Fig. 2. The comparison of the delineate diagrams shows that all adopted random variables $X_{1}, X_{2}$ and $X_{3}$ significantly affect the reliability of the structure. In the first case of the analysis (Fig. 2a), it can be seen that the adoption of the distributions given in version A strongly affects the reliability curves. Assuming the log-normal PDF of the variables $E$ and $A$ and the normal PDF of the variable $P$ reduces the range of the structure work. 
Tabel 2. Simulated cases (NG model)

\begin{tabular}{|c|c|c|c|c|c|}
\hline \multirow{2}{*}{ Case } & Random & \multicolumn{4}{|c|}{ PDF: $f_{x}\left(x_{i}\right)$} \\
\cline { 2 - 6 } & variable $X_{i}$ & $\mathrm{~A}$ & $\mathrm{~B}$ & $\mathrm{C}$ & $\mathrm{D}$ \\
\hline \hline \multirow{3}{*}{1} & $X_{1}$ & $\mathrm{~L}$ & $\mathrm{~N}$ & - & - \\
\cline { 2 - 6 } & $X_{2}$ & $\mathrm{~L}$ & $\mathrm{~N}$ & - & - \\
\cline { 2 - 6 } & $X_{3}$ & $\mathrm{~N}$ & $\mathrm{~L}$ & - & - \\
\hline \multirow{4}{*}{2} & $X_{1}$ (det.) & - & - & - & - \\
\cline { 2 - 6 } & $X_{2}$ & $\mathrm{~L}$ & $\mathrm{~N}$ & $\mathrm{~L}$ & $\mathrm{~N}$ \\
\cline { 2 - 6 } & $X_{3}$ & $\mathrm{~L}$ & $\mathrm{~N}$ & $\mathrm{~N}$ & $\mathrm{~L}$ \\
\hline \multirow{4}{*}{3} & $X_{1}$ & $\mathrm{~L}$ & $\mathrm{~N}$ & $\mathrm{~L}$ & $\mathrm{~N}$ \\
\cline { 2 - 6 } & $X_{2}$ (det.) & - & - & - & - \\
\cline { 2 - 6 } & $X_{3}$ & $\mathrm{~L}$ & $\mathrm{~N}$ & $\mathrm{~N}$ & $\mathrm{~L}$ \\
\hline \multirow{3}{*}{4} & $X_{1}$ & $\mathrm{~L}$ & $\mathrm{~N}$ & $\mathrm{~L}$ & $\mathrm{~N}$ \\
\cline { 2 - 6 } & $X_{2}$ & $\mathrm{~L}$ & $\mathrm{~N}$ & $\mathrm{~N}$ & $\mathrm{~L}$ \\
\cline { 2 - 6 } & $X_{3}$ (det.) & - & - & - & - \\
\hline
\end{tabular}

(a)

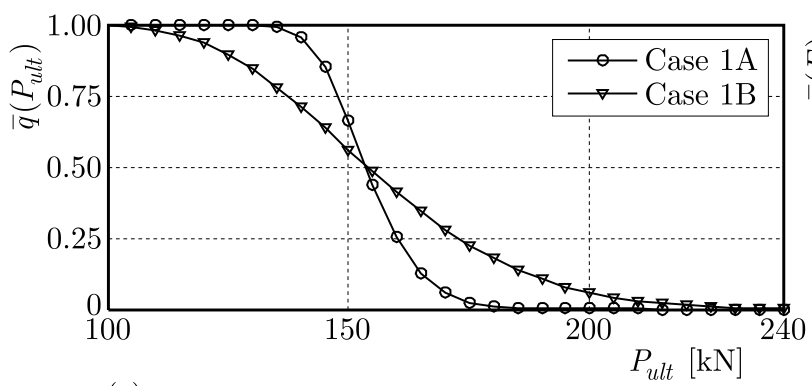

(c)

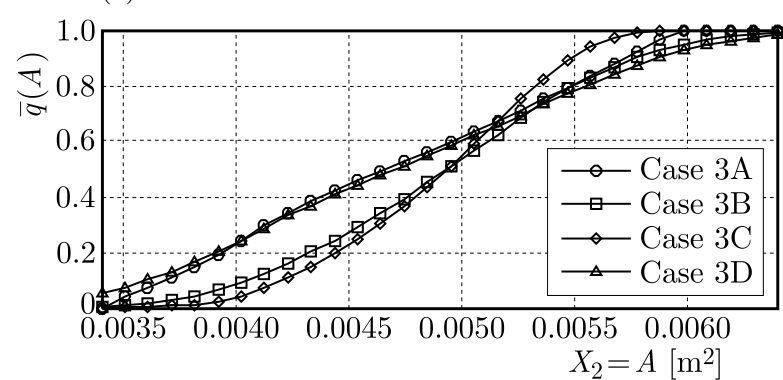

(b)

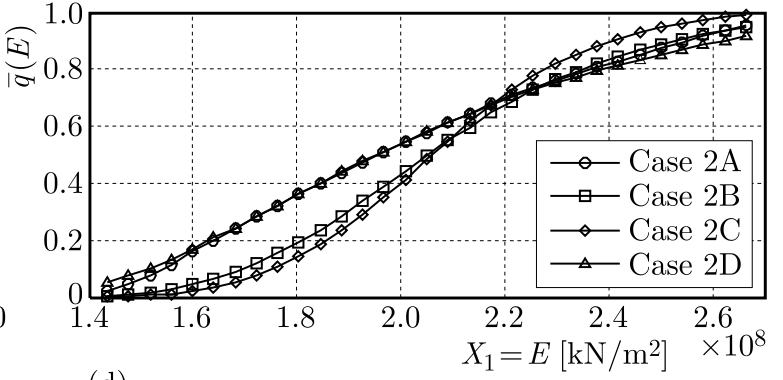

(d)

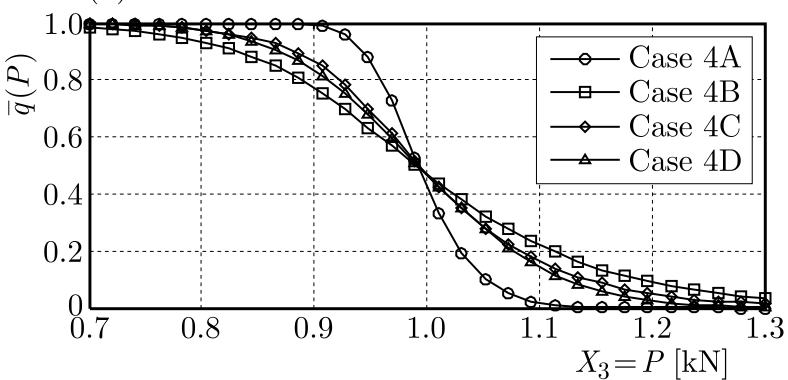

Fig. 2. Reliability curves for geometrical nonlinearity: (a) case 1, (b) case 2, (c) case 3, (d) case 4

\subsubsection{NF: physical nonlinearity, NGF: geometrical and physical nonlinearity}

Table 1 presents the mean values $\mu_{i}$ and standard deviations $\sigma_{i}$ of the assumed random variables $\mathbf{X}_{(4 \times 1)}=\left[E, A, P, \sigma_{Y}\right]$ in NG and NGF analyzes.

Table 3 contains the considered cases of reliability analysis. In case 1, it was assumed four random variables affecting the load capacity of the analyzed truss. These variables were: the modulus of elasticity $E$, the cross-sectional area of the elements $A$, the load of the nodes of the system $P$ and the yield strength of steel $\sigma_{Y}$. In cases 2-5, the value of one of the variables was treated as deterministic and adopted in its range. Reliability curves were created by performing MC simulation for each of the 30 determined variable values. In each simulation, 108 samples were drawn, separately, for each random variable according to the distribution given in Table 3 . Then, as before, by means of the formulated neural networks SWPB: $4-7-1$ and SWPB: $4-10-1$ the limit load values were determined, on the basis of which the indicator function, probability of failure and then the reliability of the structure were determined. 
Tabel 3. Simulated cases (NF and NGF models)

\begin{tabular}{|c|c|c|c|c|c|}
\hline \multirow{2}{*}{ Case } & Random & \multicolumn{4}{|c|}{ PDF: $f\left(X_{i}\right)$} \\
\cline { 3 - 6 } & variable $X_{i}$ & $\mathrm{~A}$ & $\mathrm{~B}$ & $\mathrm{C}$ & $\mathrm{D}$ \\
\hline \hline \multirow{4}{*}{1} & $X_{1}$ & $\mathrm{~L}$ & $\mathrm{~N}$ & - & - \\
\cline { 2 - 6 } & $X_{2}$ & $\mathrm{~L}$ & $\mathrm{~N}$ & - & - \\
\cline { 2 - 6 } & $X_{3}$ & $\mathrm{~N}$ & $\mathrm{~L}$ & - & - \\
\cline { 2 - 6 } & $X_{4}$ & $\mathrm{~L}$ & $\mathrm{~N}$ & - & - \\
\hline \multirow{4}{*}{2} & $X_{1}$ (det.) & - & - & - & - \\
\cline { 2 - 6 } & $X_{2}$ & $\mathrm{~L}$ & $\mathrm{~N}$ & $\mathrm{~L}$ & $\mathrm{~N}$ \\
\cline { 2 - 6 } & $X_{3}$ & $\mathrm{~L}$ & $\mathrm{~N}$ & $\mathrm{~N}$ & $\mathrm{~L}$ \\
\cline { 2 - 6 } & $X_{4}$ & $\mathrm{~L}$ & $\mathrm{~N}$ & $\mathrm{~L}$ & $\mathrm{~N}$ \\
\hline \multirow{4}{*}{4} & $X_{1}$ & $\mathrm{~L}$ & $\mathrm{~N}$ & $\mathrm{~L}$ & $\mathrm{~N}$ \\
\cline { 2 - 6 } & $X_{2}$ (det.) & - & - & - & - \\
\cline { 2 - 6 } & $X_{3}$ & $\mathrm{~L}$ & $\mathrm{~N}$ & $\mathrm{~N}$ & $\mathrm{~L}$ \\
\cline { 2 - 6 } & $X_{4}$ & $\mathrm{~L}$ & $\mathrm{~N}$ & $\mathrm{~L}$ & $\mathrm{~N}$ \\
\hline \multirow{4}{*}{4} & $X_{1}$ & $\mathrm{~L}$ & $\mathrm{~N}$ & $\mathrm{~L}$ & $\mathrm{~N}$ \\
\cline { 2 - 6 } & $X_{2}$ & $\mathrm{~L}$ & $\mathrm{~N}$ & $\mathrm{~N}$ & $\mathrm{~L}$ \\
\cline { 2 - 6 } & $X_{3}($ det.) & - & - & - & - \\
\cline { 2 - 6 } & $X_{4}$ & $\mathrm{~L}$ & $\mathrm{~N}$ & $\mathrm{~L}$ & $\mathrm{~N}$ \\
\hline \multirow{4}{*}{5} & $X_{1}$ & $\mathrm{~L}$ & $\mathrm{~N}$ & $\mathrm{~L}$ & $\mathrm{~N}$ \\
\cline { 2 - 6 } & $X_{2}$ & $\mathrm{~L}$ & $\mathrm{~N}$ & $\mathrm{~N}$ & $\mathrm{~L}$ \\
\cline { 2 - 6 } & $X_{3}$ & $\mathrm{~L}$ & $\mathrm{~N}$ & $\mathrm{~L}$ & $\mathrm{~N}$ \\
\cline { 2 - 6 } & $X_{4}($ det.) & - & - & - & - \\
\hline
\end{tabular}

Additionally, in each case, four versions (A, B, C, D) of Monte Carlo simulations differing in the adopted distribution functions of the selected random variable were considered. In total, for each type of nonlinearity, 18 reliability curves were determined for various vectors of the random variables $\mathbf{X}$. The curves are presented in Fig. 3 .

When analyzing case 1 (Fig. 3a), it can be seen that the variant A of the probability distribution function reduces the value of the load limit NF: $P_{u l t}(1 \mathrm{~A})=79 \% P_{u l t}(1 \mathrm{~B})$, NGF: $P_{u l t}(1 \mathrm{~A})=82 \% P_{u l t}(1 \mathrm{~B})$.

After analyzing the results of computations, it turned out that in the case of assuming physical nonlinearities, Young's modulus $E$ has practically no effect on the probability of structure failure. However, if physical and geometrical nonlinearities are adopted, the structure is sensitive to each of the random parameters adopted for the task.

Based on the analysis of the case of diagrams 3-5, presented in Fig. 3, it can be concluded that the random variables $X_{2}, X_{3}, X_{4}$ affect the structure reliability. Reliability curves have a smoother course for cases 3 and 5 than for case 4 . This means that the cross-section $A$ and material parameters $\sigma_{Y}$ have a smaller impact on the probability of spatial truss failure than the $P$ load.

The change in reliability depending on the adopted types of probability distributions of random variables was also analyzed. The most adverse cases are: $1 \mathrm{~A}, 3 \mathrm{C}, 4 \mathrm{~A}$ and $5 \mathrm{D}$, in which the range of work of the structure is the smallest.

\subsection{Reliability analysis of a spatial truss II}

The second example concerns the reliability analysis of a steel truss structure (Fig. 4a) susceptible to loss of stability through snap-through of the node. The layout diagram was obtained from the work by Dudzik (2016). The structure was designed from RO 135x5 pipe profiles. 
(a)
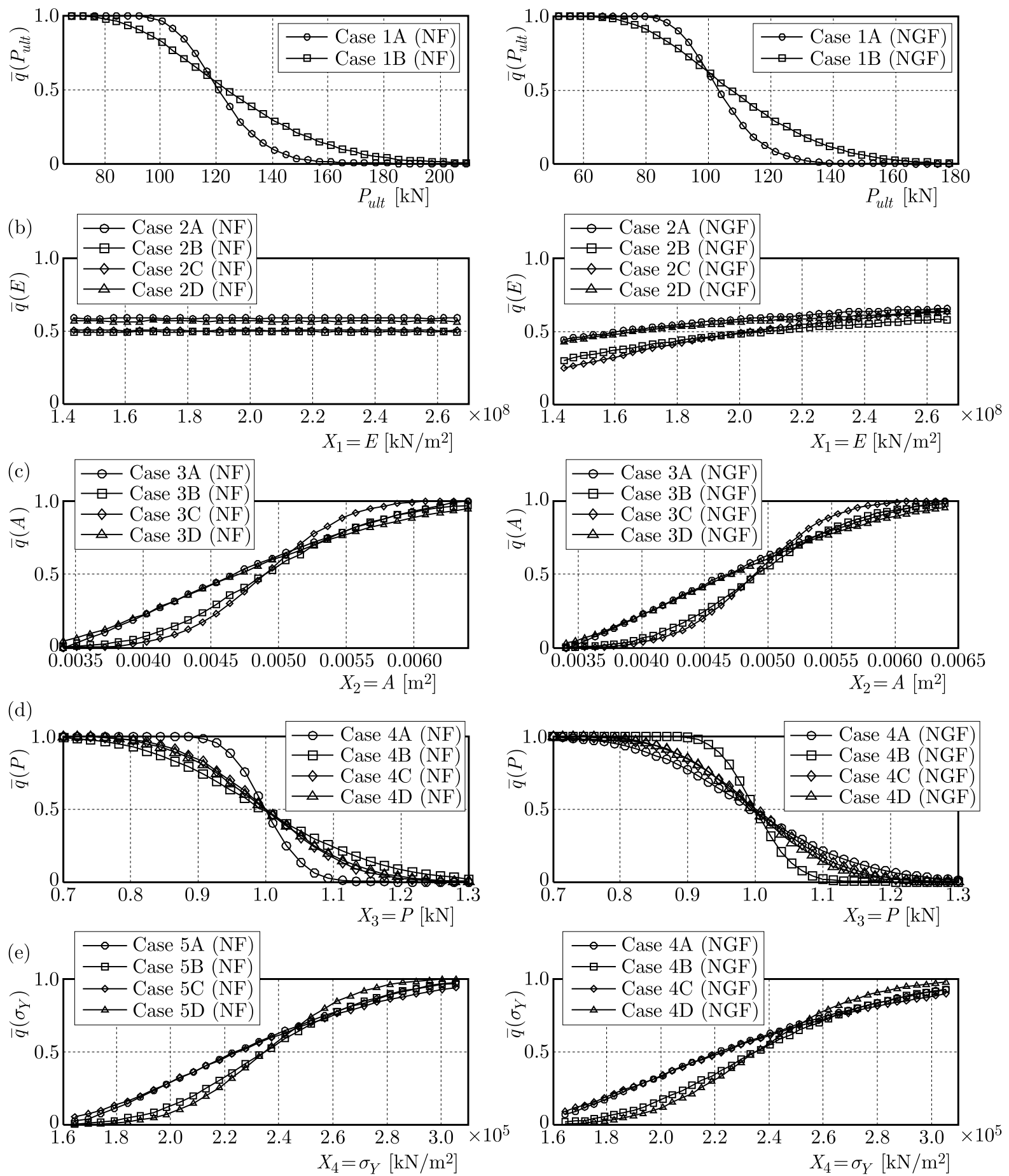

Fig. 3. Reliability curves for physical (NF) and physical and geometrical (NGF) nonlinearities: (a (a) case $1,(\mathrm{~b})$ case $2,(\mathrm{c})$ case $3,(\mathrm{~d})$ case 4 , (e) case 5

All elements are made of S355NH steel with a yield strength $\sigma_{Y}=355 \mathrm{MPa}$ and a modulus of elasticity $E=210 \mathrm{GPa}$. The support conditions were adopted in the form of a pinned fixture.

Figure $4 \mathrm{~b}$ shows examples of equilibrium paths $P\left(w_{1}\right)$ determined for vertical displacement $w_{1}$ of node 1 of the truss. The coordinates of the limit points, at the moment when the 

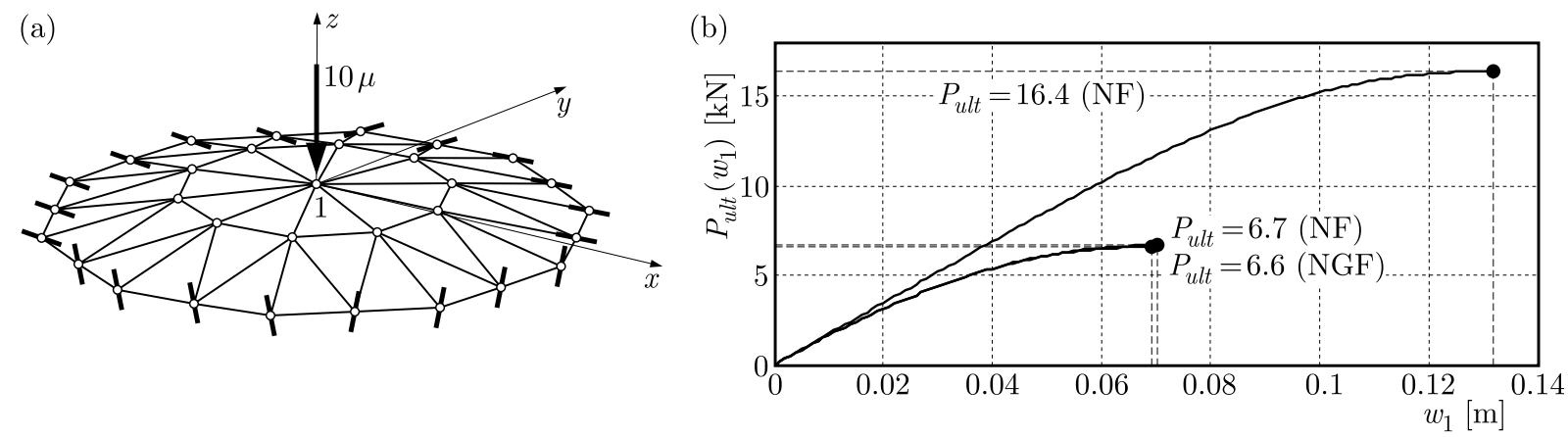

Fig. 4. (a) Spatial truss II: geometry and load diagram, (b) equilibrium paths determined for the vertical displacement $w_{1}$ of node 1 spatial truss, assuming average values of random variables

phenomenon snap-through of the node occurs, are different depending on the type of analysis adopted (NG, NF, NGF). These coordinates are:

$$
\begin{aligned}
& \mathrm{NG}-w_{1}^{G}=0.070 \mathrm{~m}, P_{u l t}=6.66 \mathrm{kN}, \\
& \mathrm{NF}-w_{1}^{G}=0.132 \mathrm{~m}, P_{u l t}=16.36 \mathrm{kN}, \\
& \mathrm{NGF}-w_{1}^{G}=0.069 \mathrm{~m}, P_{\text {ult }}=6.58 \mathrm{kN} .
\end{aligned}
$$

In this case, reaching the limit point on the equilibrium path corresponds to the global loss of stability of the structure.

Table 4 presents expected values $\mu_{i}$ and standard deviations $\sigma_{i}$ of the adopted random variables $\mathbf{X}=\left[E, A, P, \sigma_{Y}\right]$ along with the assignment of variables to the type of analysis (NG, $\mathrm{NF}, \mathrm{NGF}$ ). In this example, LN - log-normal distribution was used for material parameters $\left(E, A, \sigma_{Y}\right)$, while for the load $P$ the normal distribution $(\mathrm{N})$ was used.

Tabel 4. Description of variables and assignment to the type of analysis

\begin{tabular}{|c|c|c|c|}
\hline Random variable $X_{i}$ & Mean value $\mu_{i}$ & Standard deviation $\sigma_{i}$ & Analysis type \\
\hline \hline$X_{1}=E$ & $210 \mathrm{GPa}$ & $21 \mathrm{GPa}$ & NG+NGF \\
\hline$X_{2}=A$ & $20.42 \cdot 10^{-4} \mathrm{~m}^{2}$ & $1.02 \cdot 10^{-4} \mathrm{~m}^{2}$ & NG+NF+NGF \\
\hline$X_{3}=P$ & $10 \mathrm{kN}$ & $1 \mathrm{kN}$ & NG+NF+NGF \\
\hline$X_{4}=\sigma_{Y}$ & $355 \mathrm{MPa}$ & $7.1 \mathrm{MPa}$ & $\mathrm{NF}+\mathrm{NGF}$ \\
\hline
\end{tabular}

In this example, feedforward PBNN networks with one hidden layer, assuming the $N-H-1$ structure, were also designed (2.3). The entrance to the network were the following vectors:

$$
\begin{aligned}
& \mathrm{NG}-N=3, H=5, \mathbf{X}_{(3 \times 1)}=[E, A, P], \\
& \mathrm{NF}-N=3, H=7, \mathbf{X}_{(3 \times 1)}=\left[A, P, \sigma_{Y}\right], \\
& \mathrm{NGF}-N=4, H=10, \mathbf{X}_{(4 \times 1)}=\left[E, A, P, \sigma_{Y}\right] .
\end{aligned}
$$

Table 5 shows all cases of performed analysis. In each Monte Carlo simulation, 108 random samples were used, generated using the developed NNs, in accordance with the adopted probability density distributions.

Based on the analysis of the reliability curves presented in Fig. 5a, referring to cases 1, 2 and 3 , it can be concluded that the impact of physical nonlinearities while taking into account geometrical nonlinearities is negligibly small and amounts to approximately $0.75 \%$ for $\bar{q}\left(P_{u l t}\right)=0.95$.

In the second analyzed case, Fig. 5b, presenting the influence of the yield strength on the reliability of truss II, it can be seen that for NGF nonlinearity the parameter $\sigma_{Y}$ has no effect on 
Tabel 5. Simulated cases for example II

\begin{tabular}{|c|c|c|c|c|}
\hline \multicolumn{2}{|c|}{ Case } & $\begin{array}{c}\text { Analysis } \\
\text { type }\end{array}$ & $\begin{array}{c}\text { Random } \\
\text { variable } X_{i}\end{array}$ & $\begin{array}{c}\text { Determined } \\
\text { variables }\end{array}$ \\
\hline \multicolumn{2}{|c|}{$\overline{1} 1$} & $\overline{N G}$ & $X_{1}, X_{2}, X_{3}$ & - \\
\hline \multicolumn{2}{|r|}{2} & $\mathrm{NF}$ & $X_{2}, X_{3}, X_{4}$ & - \\
\hline \multicolumn{2}{|r|}{3} & NGF & $X_{1}, X_{2}, X_{3}, X_{4}$ & - \\
\hline \multirow{2}{*}{4} & $\mathrm{~A}$ & $\mathrm{NF}$ & $X_{2}, X_{3}$ & \multirow{2}{*}{$X_{4}=\sigma_{Y}$} \\
\hline & $\bar{B}$ & NGF & $X_{2}, X_{3}, X_{4}$ & \\
\hline \multirow{3}{*}{5} & $\mathrm{~A}$ & $\mathrm{NG}$ & $X_{1}, X_{3}$ & \multirow{3}{*}{$X_{2}=A$} \\
\hline & $\bar{B}$ & $\mathrm{NF}$ & $X_{3}, X_{4}$ & \\
\hline & $\mathrm{C}$ & NGF & $X_{1}, X_{3}, X_{4}$ & \\
\hline \multirow{3}{*}{6} & $\mathrm{~A}$ & $\mathrm{NG}$ & $X_{1}, X_{2}$ & \multirow{3}{*}{$X_{3}=P$} \\
\hline & $\bar{B}$ & $\mathrm{NF}$ & $X_{2}, X_{4}$ & \\
\hline & $\mathrm{C}$ & NGF & $X_{1}, X_{2}, X_{4}$ & \\
\hline \multirow{2}{*}{7} & $\mathrm{~A}$ & $\mathrm{NG}$ & $X_{2}, X_{3}$ & \multirow{2}{*}{$X_{1}=E$} \\
\hline & $\mathrm{B}$ & NGF & $X_{2}, X_{3}, X_{4}$ & \\
\hline
\end{tabular}

the work of the structure, while during the NF analysis the yield strength value is important in the reliability analysis. Figures $5 \mathrm{c}$,d,e show successive cases 5-7, showing the impact of the determined values of the parameters (cross-sectional area $A$, load values $P$ and Young's modulus $E$ ) on the shape of reliability curves.

The graphs above show that for all types of nonlinearity (NG, NF and NGF), the parameters $A$ and $E$ and load $P$ play an important and similar role in the reliability analysis.

\section{Summary and general conclusions}

The article presents the use of neural networks to analyze the reliability of spatial trusses taking into account physical and geometrical nonlinearities. Computations were made using a hybrid system. This system was created by working sequentially, two separate FEM and NN applications. Using the FEM program, sets for learning and testing the neural networks were generated, then PBNN networks were used to generate samples in simulations of the classic Monte Carlo method to determine reliability curves. The reliability curves can be used in the engineering design process because they show changes in structure reliability caused by fluctuations of selected design parameters that are random.

Analyzis of the reliability of the first spatial truss with taking into account the material nonlinearity significantly reduced the safe range of operation of the structure, but did not change the reliability curve of the Lamella dome (example II).

Comparison of the computed reliability curves shows that if only physical nonlinearities are assumed in the structure, Young's modulus practically has no effect on the probability of structure failure. The significant impact of other task parameters, i.e. cross-sectional area, yield strength and the value of the applied load on the reliability is similar in all tasks analyzed in this work.

It also turned out that the reliability of the structure is largely influenced by the adopted functions of probability distribution of random variables. The most unfavorable cases in which the range of working of the structure is the smallest (reliability reaches zero value faster than for other variants) occur when a log-normal distribution is used for material parameters, whereas the normal distribution is used for the load. 
(a)

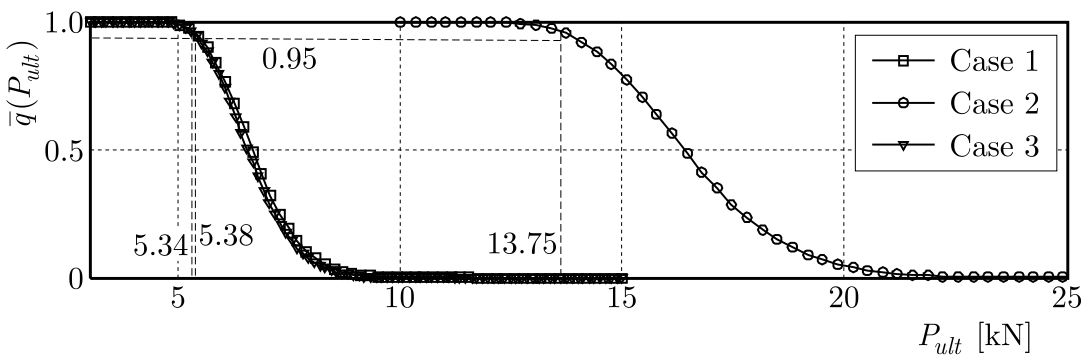

(b)

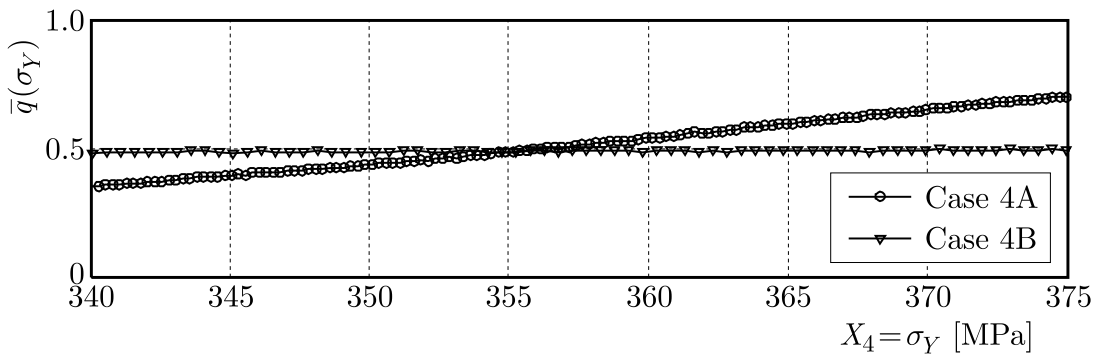

(c)

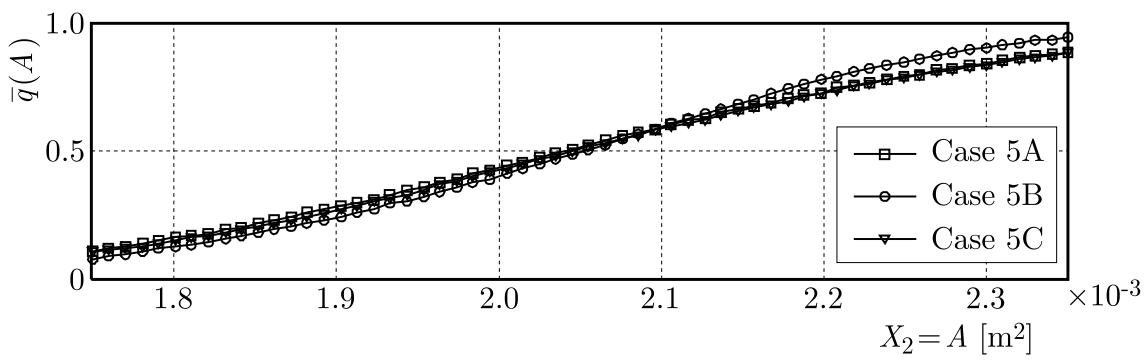

(d)

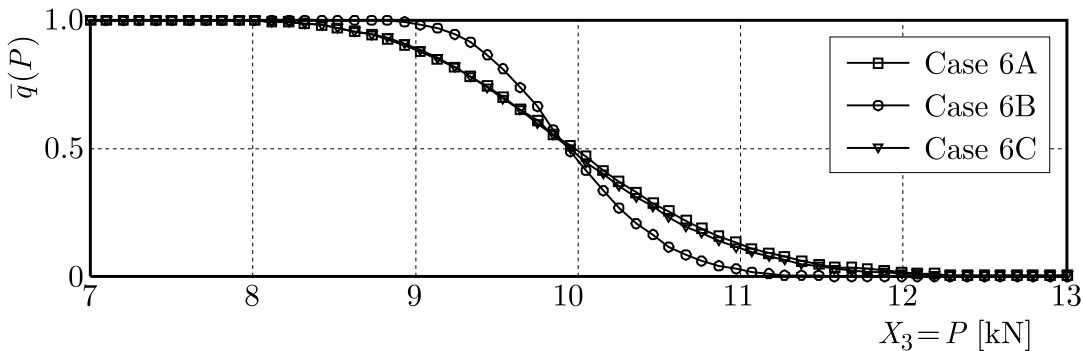

(e)

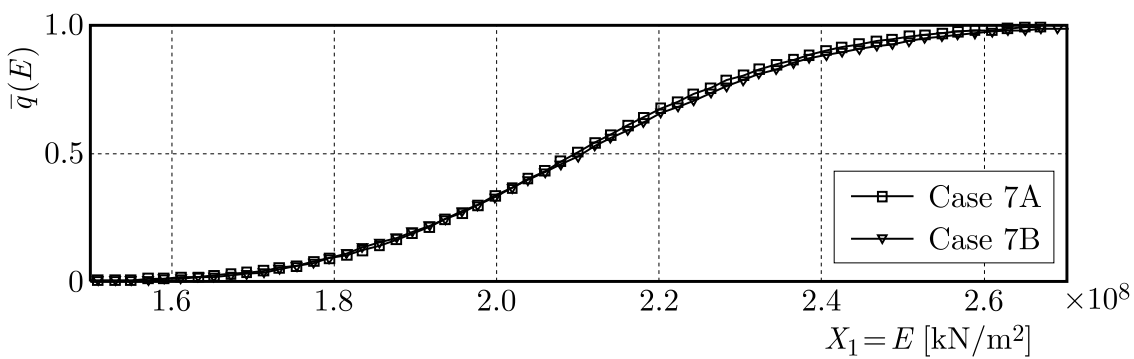

Fig. 5. Reliability curves: (a) cases $1-3$, (b) case 4, (c) case 5, (d) case 6, (e) case 7

\section{References}

1. Carpentier A., Munos R., 2012, Adaptive stratified sampling for Monte-Carlo integration of differentiable functions, Proceedings of the 25th International Conference on Neural Information Processing Systems, 1

2. Deng J., Gu D., Li X., Yue Z.Q., 2005, Structural reliability analysis for implicit performacne functions using neural networks. Structural Safety, 27, 25-48, DOI: 10.1016/j.strusafe.2004.03.004

3. Ditlevsen O., Madsen H., 1996, Structural Reliability Methods, Wiley \& Sons 
4. DudzIK A., 2016, The effectiveness of the FORM method compared to other probabilistic methods in the analysis of bar structures, PHD Disertation (in Polish), Politechnika Świętokrzyska, Kielce.

5. DuDZIK A., 2017, Reliability assessment of steel-aluminium lattice tower, IOP Conference Series: Materials Science and Engineering, 245, 1-9, DOI: 10.1088/1757-899X/245/3/032072

6. Dudzik A., Potrzeszcz-Sut B., 2019, The structural reliability analysis using explicit neural state functions, MATEC Web of Conferences, 262, 1, DOI: 10.1051/matecconf/201926210002

7. GwóźDź M., Machowski A., 2011, Selected Tests and Calculations of Building Constructions by Probabilistic Methods (in Polish), Wydawnictwo Politechniki Krakowskiej im. Tadeusza Kościuszki, Kraków

8. Hagan M., Menhaj M., 1994, Training feedforward networks with the Marquardt algorithm, IEEE Transactions on Neural Networks, 5, 6, 989-993, DOI: 10.1109/72.329697

9. HaYkin S., 1999, Neural Networks - A Comprehensive Foundation, Prentice Hall, New York

10. JCSS, 1981, Joint committee on structural safety, general principles on reliability for structural design, IABSE

11. Jelic A., Baitsch M., Hartmann D., Spitzlei K., Ballnus D., 2004, Distributed computing of failure probabilities for structures in civil engineering, X International Conference on Computing in Civil and Building Engineering, Weimar

12. Kaliszuk J., 2011, Hybrid Monte Carlo method in the reliability analysis of structures, Computer Assisted Mechanics and Engineering Sciences, 18, 205-216

13. Kaliszuk J., Waszczyszyn Z., 2006, Reliability analysis of a steel girder by the hybrid Monte Carlo method. Progress in steel, composite and aluminium structures, Proceedings of the XIth International Conference on Metal Structures, Rzeszów, 843-847

14. Knauff M., 2015, Calculation of Reinforced Concrete Structures According to Eurocode 2 (in Polish), PWN, Warszawa

15. Kunstmann H., Kinzelbach W., Siegfried T., 2002, Conditional first-order second-moment method and its application to the quantification of uncertainty in groundwater modeling, Water Resources Reaserch, 38, 4-1035, 6.1-6.14

16. Masters T., 1993, Practical Neural Network Recipies in $C++$, Morgan Kaufmann

17. Metropolis N., Ulam S., 1949, The Monte Carlo Method, Journal of the American Statistical Association, 44, 247, 335-341, DOI: 10.1029/2000WR000022

18. Olofsson P., Andersson M., 2012, Probability, Statistics, and Stochastic Processes, 2nd ed., Wiley-Interscience, DOI: 10.1002/9781118231296.ch3

19. PABISEK E., 2008, Hybrid Systems Integrating FEM and SSN in the Analysis of Selected Problems of Structural Mechanics and Materials (in Polish), Politechnika Krakowska, Kraków

20. Pabisek E., Kaliszuk J., Waszczyszyn Z., 2004, Neural and finite element analysis of a plane steel frame reliability by the Classical Monte Carlo method, Artificial Intelligence and Soft Computing - ICAISC 2004, 7th International Conference, Zakopane, 1081-1086

21. Papadopoulos V., Giovanis D., Lagaros N., Papadrakakis M., 2012, Accelerated subset simulation with neural networks for reliability analysis, Computer Methods in Applied Mechanics and Engineering, 223-224, 70-80, DOI: 10.1016/j.cma.2012.02.013

22. Papadrakakis M., Papadopoulos V., Lagaros N., 1996, Structural reliability analysis of elastic-plastic structures using neural networks and Monte Carlo simulation, Computer Methods in Applied Mechanics and Engineering, 136, 145-163, DOI: 10.1016/0045-7825(96)01011-0

23. PN-EN 1990:2004, Basics of structural design (in Polish), PKN, Warszawa

24. PN-EN 1993-1-1:2006, Steel structure design - Part 1-1: General rules and rules for buildings (in Polish), PKN, Warszawa 
25. PN-EN 1993-1-4:2007, Designing of steel structures - Part 1-4: General rules - Supplementary rules for stainless steel structure (in Polish), PKN, Warszawa

26. Radoń U., 2012, Application of the FORM Method in the Analysis of Reliability of Truss Structures Susceptible Node Snapping (in Polish), Wydawnictwo Politechniki Świętokrzyskiej, Kielce

27. Ramberg W., Osgood W., 1943, Description of stress-strain curves by three parameters, Technical Note No. 902, National Committee for Aeronautics, Washington DC

28. Rubinstein R., Kroese D., 2008, Simulation and the Monte Carlo Method, Wiley-Interscience, DOI: $10.1002 / 9781118631980$

29. Tsompanakis Y., Lagaros N., Stavroulaki G.E., 2005, Efficient neural network models for structural reliability analysis and identification problems, The Eighth International Conference on the Application of Artificial Intelligence to Civil, Structural and Environmental Engineering, DOI: $10.4203 / \operatorname{ccp} .82 .41$

30. Waszczyszyn Z., Cichoń C., Radwańska M., 1994, Stability of Structures by Finite Element Methods, Elsevier, Amsterdam 